\title{
Switched system modeling and robust steering control of the tail end phase in a hot strip mill
}

\author{
Ivan Malloci ${ }^{\mathrm{a}}$, Jamal Daafouz ${ }^{*, a}$, Claude Iung $^{\mathrm{a}}$, Rémi Bonidal ${ }^{\mathrm{b}}$, \\ Patrick Szczepanski ${ }^{b}$ \\ ${ }^{a}$ Centre de Recherche en Automatique de Nancy, UMR 7039 CNRS - Nancy Université, \\ ENSEM, 2, Avenue de la forêt de Haye 54516 Vandoeuvre-lès-Nancy, France \\ ${ }^{b}$ ArcelorMittal Maizières, RED Industrial Operations, BP 30320, F-57283 \\ Maizières-lès-Metz Cedex, France
}

\begin{abstract}
In this article, a robust steering control for the last phase of the rolling process in a hot strip mill is proposed. This phase, called tail end phase, may be modelled as a linear switched system. The switchings make the system unstable and the task of the tail end steering control consists in guaranteeing the safety of the industrial plant. The system involves a two time scales dynamics. Hence, the singular perturbation method is used in order to design the control law. The controller has to take into account the physical variations of the rolled products and an uncertainty in the switching time. Results concerning the ArcelorMittal hot strip mill of Eisenhüttenstadt are presented.
\end{abstract}

Key words: Robust Steering Control, Polytopic Uncertainties, Switched Systems, Singular Perturbations, Linear Matrix Inequalities.

\section{Introduction}

In the steel production framework, the steering control denotes the strategies to guide a metal strip in a finishing mill, which is constituted by $n$ stands. Each stand contains one set of rolls, in order to crush the strip. In the hot rolling process, the thickness of a strip is reduced from several tens of millimeters to fewer millimeters. The task consists in obtaining a strip with

\footnotetext{
*Corresponding author:

Email address: Jamal.Daafouz@ensem.inpl-nancy.fr (Jamal Daafouz)
} 
constant thickness, guaranteeing the product quality and the system safety. This goal is reached by maintaining the strip in a straight line and close to the mill axis, avoiding sudden lateral movements of the rolled product. The lateral displacement of the strip is called strip off-centre.

In last few years, several steering control methods have been developed. In general, the strip off-centre of each stand is considered the differential force image of the same stand. Then, different approaches have been proposed in order to compute the stand tilting: PID controllers [4], [9], [11], [18], [25], optimal regulators [24], state-feedback poles assignment [21], coefficient diagram methods (CDM) [17], sliding mode techniques [20] and model predictive control (MPC) [2]. Nevertheless, the law linking the differential force and the strip off-centre is strongly non-linear. Moreover, each stand is linked to the others by the strip traction. These constraints are taken into account in [3], where a LQ controller is designed for a nominal framework, and in [16], where a $H_{2}$ robust control is proposed. These last two approaches treat only the first phase of the rolling process, called $n$-stands phase. In this phase, the strip is connected to all the stands and the main control task consists in guaranteeing a good quality of the rolled product.

The purpose of this article is the design of a robust steering control for the last phase of the rolling process, the tail end phase. In this phase, the strip leaves the stands one after the other. Each time the strip leaves a stand, the system dynamics changes. A system with this behaviour can be defined as a switched system [12]. The loss of traction due to the switchings makes the system unstable. It is in this phase that a crash can occur, damaging the rolls. Hence, the aim of the tail end steering control consists in guaranteeing the system stability and the safety of the rolling process. A mill treats products with very different characteristics. Then, the variation of the physical parameters has to be taken into account in the control design. Also an uncertainty in the switching signal is considered. Moreover, the system involves a two time scales dynamics. In this case, standard control methods can lead to ill-conditioning controllers. To avoid numerical problems, singular perturbation methods can be used: the system dynamics is decomposed into fast and slow manifolds and a different controller is designed for each of them [10]. LMI techniques are used in order to design the control law [1].

To the best of our knowledge, this is the first article which proposes a tail end steering control taking into account the physical relations linking the stands, the effects of the switchings on the system dynamics and the fact that the system treats products with different characteristics. 
The article is organised as follows. In section 2, the physical description of the system is given. In section 3, a model of the tail end phase as an uncertain linear switched system in the singular perturbation form is proposed. In section 4 the control design is presented. In section 5 , results concerning the ArcelorMittal hot strip mill of Eisenhüttenstadt (Germany) are presented.

The following notations are used: for a matrix $X, X^{\prime}$ denotes its transpose and $\operatorname{Tr}(X)$ its trace. $X \succ 0(X \succeq 0)$ indicates that $X$ is positive (no negative) definite. $X$ is said to be Hurwitz if all its eigenvalues have negative real parts. Furthermore, $X$ is said to be Schur if all its eigenvalues have modulus smaller than one. $\varphi\{X\}$ denotes the spectrum of $X$ and the symbol $(\star)^{\prime}$ denotes a symmetric block. $I_{n}$ indicates the identity matrix $\in \mathbb{R}^{n \times n}$.

\section{Hot strip mill physical description}

A hot strip mill (HSM) is constituted by $n$ stands. Each stand contains one set of rolls (composed of two work rolls and two support rolls) and the strip in the inter-stand on the front. In the ArcelorMittal HSMs $n=\{5,6,7\}$. Since a HSM treats products with different characteristics, an uncertainty has to be considered for the physical parameters. A polytopic approach will be used in order to describe these uncertainties. $\nu \in \Gamma=\left\{1, \ldots, \mathcal{N}_{v}\right\}$ denotes the vertices of the resulting convex hull. $\mathcal{N}_{v}$ is the number of uncertain parameters.

For each stand $j \in \Upsilon=\{1, \ldots, n\}$, the main physical parameters are the strip width $w_{j, \nu}$, the strip thickness $h_{j, \nu}$, the back strip tension $T_{j, \nu}^{a m}$, the front strip tension $T_{j, \nu}^{a v}$, the screw interaxis length $l_{j, \nu}^{v}$, the interstand length $l_{j, \nu}^{0}$, the work rolls length $b_{j}$, the work rolls speed $s_{j, \nu}$ and the Young's module $E_{j, \nu}$. Also the following constants are necessary to completely define a strip: $c_{j, \nu}^{f h}, c_{j, \nu}^{f T_{a m}}, c_{j, \nu}^{f T_{a v}}, c_{j, \nu}^{g h}, c_{j, \nu}^{g T_{a m}}, c_{j, \nu}^{g T_{a v}}, K_{j, \nu}^{h}, K_{j, \nu}^{f}, K_{j, \nu}^{l}, P_{j, \nu}$ and $g_{j, \nu}$. The main asymmetries are the strip off-centre $Z_{j}$, the strip thickness profile (wedge) $\Delta h_{j}$, the stand tilting $\Delta S_{j}$, the differential stand stretch $\Delta K_{j}$, the differential rolling force $\Delta P_{j}$, the upstream differential of strip tension $\Delta T_{j}^{a m}$ and the downstream differential of strip tension $\Delta T_{j}^{a v}$.

As long as the strip remains connected to the coilbox, which is the device used to coil the strips into the finishing train, the HSM model does not change (Fig. 1). Otherwise, in the last phase of the rolling process, the tail end phase, the strip leaves the stands, one after the other. Each time the strip leaves a stand the system dynamics changes. Then, the HSM can be defined as a switched system. The first subsystem (the strip has not yet left 


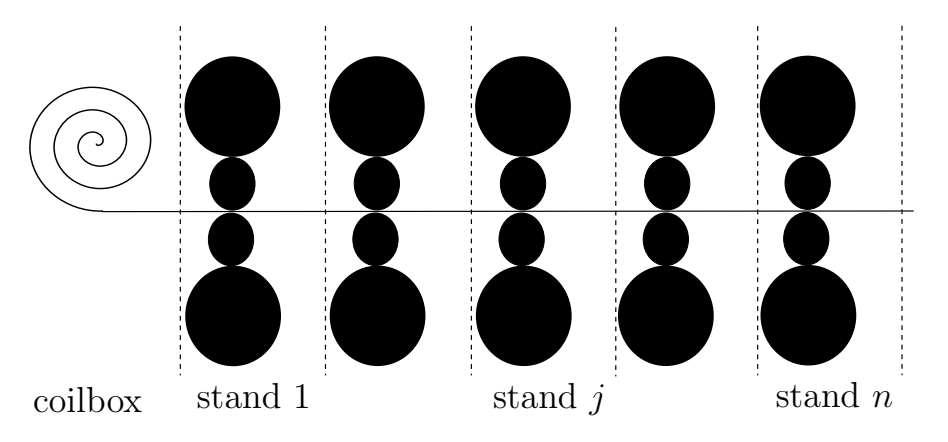

Figure 1: HSM lateral view

the first stand) is called $n$-stands subsystem. The subsystem active after the $i^{\text {th }}$ switching, which occurs when the strip leaves the $i^{\text {th }}$ stand, is called $(n-i)$-stands subsystem. The following main equations, which are relevant for $j>i$, govern the system:

- The differential rolling force equation:

$$
\Delta P_{j}=c_{j-1, \nu}^{f h} \Delta h_{j-1}+c_{j, \nu}^{f h} \Delta h_{j}+c_{j, \nu}^{f T_{a m}} \Delta T_{j}^{a m}+c_{j, \nu}^{f T_{a v}} \Delta T_{j}^{a v}
$$

- The exit stand wedge equation:

$$
\begin{aligned}
\Delta h_{j}= & \left(\frac{w_{j, \nu}}{\left(l_{j, \nu}^{v}\right)^{2} K_{j, \nu}^{h}}+\frac{6 w_{j, \nu}}{b_{j, \nu}^{2} K_{j, \nu}^{f}}\right)\left(\Delta P_{j}+2 P_{j, \nu}\right) Z_{j}+ \\
& \frac{\Delta P_{j}}{K_{j, \nu}^{l}}+\frac{w_{j, \nu}}{l_{j, \nu}^{v}} \Delta S_{j}-\frac{w_{j, \nu}}{l_{j, \nu}^{v}\left(K_{j, \nu}^{h}\right)^{2}} P_{j, \nu} \Delta K_{j}
\end{aligned}
$$

- The angle $\alpha_{j}$ between the strip and the mill axis equation:

$$
\begin{aligned}
\dot{\alpha}_{j}= & \frac{s_{j, \nu}}{w_{j, \nu}}\left(\frac{c_{j, \nu}^{g h}}{1+g_{j, \nu}}+\frac{1}{h_{j, \nu}}\right) \Delta h_{j}+\frac{s_{j, \nu}}{w_{j, \nu}}\left(\frac{c_{j-1, \nu}^{g h}}{1+g_{j, \nu}}-\frac{1}{h_{j-1, \nu}}\right) \Delta h_{j-1}+ \\
& \frac{s_{j, \nu}}{w_{j, \nu}} \frac{c_{j, \nu}^{g T_{a v}}}{\left(1+g_{j, \nu}\right)} \Delta T_{j}^{a v}+\frac{s_{j, \nu}}{w_{j, \nu}} \frac{c_{j, \nu}^{g T_{a m}}}{\left(1+g_{j, \nu}\right)} \Delta T_{j}^{a m}
\end{aligned}
$$

- The strip off-centre equation:

$$
\dot{Z}_{j}=s_{j, \nu} \alpha_{j}
$$

Moreover, for $j>i+1$ we have: 
- The upstream differential of strip tension equation:

$$
\begin{aligned}
\Delta T_{j}^{a m}= & 3\left(\frac{w_{j, \nu} E_{j, \nu}}{\left(l_{j, \nu}^{0}\right)^{2}}+\frac{T_{j, \nu}^{a m}}{w_{j, \nu}}\right)\left(Z_{j}-Z_{j-1}\right)+ \\
& \frac{w_{j, \nu} E_{j, \nu}}{l_{j, \nu}^{0}}\left(2 \alpha_{j}-\alpha_{j-1}\right)+3 \frac{l_{j, \nu}^{0} T_{j, \nu}^{a m}}{w_{j, \nu}} \alpha_{j} ;
\end{aligned}
$$

- The coupling between two successive stands equation:

$$
\Delta T_{j-1}^{a v}=-\Delta T_{j}^{a m}
$$

For the last two equations, there exists an exception. When the $n$-stands subsystem is on, the equations (5) and (6) are verified $\forall j \in \Upsilon$. In this case, the upstream differential of strip tension in the first stand $\Delta T_{1}^{a m}$ can take two different values. It corresponds to the downstream tension of the coilbox $\Delta T_{1}^{a m}=-\Delta T_{0}^{a v}$ when the strip is connected to the coilbox (most of the time), and to zero after the strip leaves the coilbox. This last phase with $\Delta T_{1}^{a m}=0$ and $\Delta T_{2}^{a m} \neq 0$ (the strip left the coilbox but not yet the first stand) has not been considered in the switching system model because is very short and its dynamics is similar to the case $\Delta T_{1}^{a m}=-\Delta T_{0}^{a v}$ and $\Delta T_{2}^{a m} \neq 0$. When the strip leaves the first stand the system switches to the $(n-1)$-stands subsystem and the equations (5) and (6) are relevant for $j>i+1$.

According to the previous physical equations, the system is described by the non-linear continuous-time differential uncertain switched system

$$
\left\{\begin{array}{l}
\dot{z}=\Phi^{\sigma(t)}(z, u, \nu) \\
y=\mathcal{C}^{\sigma(t)} z
\end{array}\right.
$$

where

$$
z=\left[\alpha_{1}, \ldots, \alpha_{n}, Z_{1}, \ldots, Z_{n}\right]^{\prime} \in \mathbb{R}^{2 n}
$$

is the state, $u \in \mathbb{R}^{r}$ is the control signal (the stand tilting $\Delta S$ ), $y \in \mathbb{R}^{m}$ is the output signal, $\left\{\Phi^{\rho}: \rho \in \Sigma\right\}$ is a family of nonlinear functions, $\sigma(t): \mathbb{N} \rightarrow$ $\Sigma=\left\{1, \ldots, \mathcal{N}_{s}\right\}$ is the switching signal, which is assumed to be unknown a priori, and $\mathcal{N}_{s}$ represents the number of subsystems. $\Phi^{\sigma(t)}$ and $\mathcal{C}^{\sigma(t)}$ can be written in the polytopic form

$$
\Phi^{\sigma(t)}(z, u, \nu)=\sum_{\rho=1}^{\mathcal{N}_{s}} \xi_{\rho}(t) \hat{\phi}^{\rho}=\sum_{\rho=1}^{\mathcal{N}_{s}} \sum_{\nu=1}^{\mathcal{N}_{v}} \xi_{\rho}(t) \lambda_{\rho, \nu}(t) \phi^{\rho, \nu},
$$




$$
\mathcal{C}^{\sigma(t)}=\sum_{\rho=1}^{\mathcal{N}_{s}} \xi_{\rho}(t) \bar{C}^{\rho},
$$

with $\xi_{\rho}(t): \mathbb{N} \rightarrow\{0,1\}, \sum_{\rho=1}^{\mathcal{N}_{s}} \xi_{\rho}(t)=1, \lambda_{\rho, \nu}(t) \geq 0, \sum_{\rho=1}^{\mathcal{N}_{v}} \lambda_{\rho, \nu}(t)=1, \forall(\rho, \nu) \in$ $\Sigma \times \Gamma$.

\section{Problem formulation}

Only small deviations around the ideal operating point $(z=0)$ are assumed. Hence, for designing the control law, the following linearized model can be considered:

$$
\left\{\begin{array}{l}
\dot{z}=\mathcal{M}^{\sigma(t)} z+\mathcal{N}^{\sigma(t)} u \\
y=\mathcal{C}^{\sigma(t)} z
\end{array}\right.
$$

where

$$
\begin{aligned}
\mathcal{M}^{\sigma(t)} & =\sum_{\rho=1}^{\mathcal{N}_{s}} \xi_{\rho}(t) \hat{\mathcal{M}}^{\rho}=\sum_{\rho=1}^{\mathcal{N}_{s}} \sum_{\nu=1}^{\mathcal{N}_{v}} \xi_{\rho}(t) \lambda_{\rho, \nu}(t) \bar{M}^{\rho, \nu} \\
\mathcal{N}^{\sigma(t)} & =\sum_{\rho=1}^{\mathcal{N}_{s}} \xi_{\rho}(t) \hat{\mathcal{N}}^{\rho}=\sum_{\rho=1}^{\mathcal{N}_{s}} \sum_{\nu=1}^{\mathcal{N}_{v}} \xi_{\rho}(t) \lambda_{\rho, \nu}(t) \bar{N}^{\rho, \nu} .
\end{aligned}
$$

The pairs $\left(\bar{M}^{\rho, \nu}, \bar{N}^{\rho, \nu}\right)$ and $\left(\bar{C}^{\rho}, \bar{M}^{\rho, \nu}\right)$ are assumed to be controlable and observable $\forall(\rho, \nu) \in \Sigma \times \Gamma$, respectively.

In the HSM system, a two time scale dynamics has to be considered. Thus, standard control methods can yield to ill-conditioning controllers. The singular perturbation method can be used to avoid numerical problems. This method consists in decomposing the system dynamics into fast and slow manifolds and in designing a different controller for each of them [10]. Consider a vertex $(\rho, \nu)$ of the polytopic system (11):

$$
\left\{\begin{array}{l}
\dot{z}=\bar{M}^{\rho, \nu} z+\bar{N}^{\rho, \nu} u \\
y=\bar{C}^{\rho} z
\end{array}\right.
$$

In order to write the subsystem (14) in the singular perturbation form, the components of the state vector $z$ which belongs to the fast and the slow dynamics must be divided into two different state vectors $x_{1}$ and $x_{2}$. In the $n$-stands subsystem, the slow dynamics is given by the $n$ strip off-centre. 
In the tail end subsystems, the slow dynamics is given by the strips offcentre of the operating stands and the angle corresponding to the first active stand. Hence, the components and the dimension of $x_{1}$ and $x_{2}$ are different $\forall \rho \in \Sigma$. Nevertheless, there exists a set of permutation matrices $E^{\rho}$, with $\operatorname{det}\left(E^{\rho}\right)= \pm 1$ and $E^{\rho} E^{\rho-1}=I_{n}$, such that the change of basis

$$
x^{\rho}=E^{\rho} z
$$

yields a system state in the form:

$$
x^{\rho}=\left[\begin{array}{l}
x_{1}^{\rho} \\
x_{2}^{\rho}
\end{array}\right],
$$

with $x_{1}^{\rho} \in \mathbb{R}^{n_{1}^{\rho}}$ and $x_{2}^{\rho} \in \mathbb{R}^{n_{2}^{\rho}}, \forall \rho \in \Sigma$. Hence, the subsystem (14) can be written in the standard singular perturbation form:

$$
\left\{\begin{array}{l}
\varepsilon \dot{x}_{1}^{\rho}=M_{11}^{\rho, \nu} x_{1}^{\rho}+M_{12}^{\rho, \nu} x_{2}^{\rho}+N_{1}^{\rho, \nu} u \\
\dot{x}_{2}^{\rho}=M_{21}^{\rho, \nu} x_{1}^{\rho}+M_{22}^{\rho, \nu} x_{2}^{\rho}+N_{2}^{\rho, \nu} u \\
y=C_{1}^{\rho} x_{1}^{\rho}+C_{2}^{\rho} x_{2}^{\rho}
\end{array}\right.
$$

where $M_{11}^{\rho, \nu}$ is assumed to be Hurwitz and $\varepsilon>0$ is a scalar parameter $\ll 1$.

According to the practical implementation, the controller must be designed in the discrete-time, with a sample time of $T_{s}=0.05 \mathrm{sec}$. Then, the discrete-time form of (17) is used [8]:

$$
\left\{\begin{array}{l}
x_{1}^{\rho}(k+1)=A_{11}^{\rho, \nu} x_{1}^{\rho}(k)+A_{12}^{\rho, \nu} x_{2}^{\rho}(k)+B_{1}^{\rho, \nu} u(k) \\
x_{2}^{\rho}(k+1)=\varepsilon A_{21}^{\rho, \nu} x_{1}^{\rho}(k)+\left(I_{n_{2}}+\varepsilon A_{22}^{\rho, \nu}\right) x_{2}^{\rho}(k)+B_{2}^{\rho, \nu} u(k) \\
y(k)=C_{1}^{\rho} x_{1}^{\rho}(k)+C_{2}^{\rho} x_{2}^{\rho}(k)
\end{array}\right.
$$

Let

$$
\begin{aligned}
& A^{\rho, \nu}(\varepsilon)=\left[\begin{array}{cc}
A_{11}^{\rho, \nu} & A_{12}^{\rho, \nu} \\
\varepsilon A_{21}^{\rho, \nu} & \left(I_{n_{2}^{\rho}}+\varepsilon A_{22}^{\rho, \nu}\right)
\end{array}\right], \\
& B^{\rho, \nu}(\varepsilon)=\left[\begin{array}{c}
B_{1}^{\rho, \nu} \\
\varepsilon B_{2}^{\rho, \nu}
\end{array}\right] \\
& C^{\rho}=\left[\begin{array}{ll}
C_{1}^{\rho} & C_{2}^{\rho}
\end{array}\right] .
\end{aligned}
$$

The slow subsystem is defined as:

$$
\left\{\begin{array}{l}
x_{s}^{\rho}(k+1)=\left(I_{n_{2}^{\rho}}+\varepsilon A_{s}^{\rho, \nu}\right) x_{s}^{\rho}(k)+\varepsilon B_{s}^{\rho, \nu} u_{s}(k) \\
y_{s}(k)=C_{s}^{\rho, \nu} x_{s}^{\rho}(k)+D_{s}^{\rho, \nu} u_{s}(k)
\end{array}\right.
$$


Table 1: Eigenvalues Comparison

\begin{tabular}{|c|c|}
\hline$\varphi\left\{A^{\rho}\right\}$ & $\varphi\left\{A_{s}^{\rho}\right\}$ \\
\hline \hline 1.0198 & 1.0197 \\
\hline 0.9860 & 0.9861 \\
\hline 0.9144 & 0.9154 \\
\hline 0.8922 & 0.8933 \\
\hline 0.8120 & 0.8167 \\
\hline 0.0301 & \\
\hline $0.0028+0.0217 \mathrm{i}$ & \\
\hline $0.0028-0.0217 \mathrm{i}$ & \\
\hline
\end{tabular}

with

$$
\begin{aligned}
& A_{s}^{\rho, \nu}=A_{22}^{\rho, \nu}+A_{21}^{\rho, \nu}\left(I_{n_{1}^{\rho}}-A_{11}^{\rho, \nu}\right)^{-1} A_{12}^{\rho, \nu} \\
& B_{s}^{\rho, \nu}=B_{2}^{\rho, \nu}+A_{21}^{\rho, \nu}\left(I_{n_{1}^{\rho}}-A_{11}^{\rho, \nu}\right)^{-1} B_{1}^{\rho, \nu} \\
& C_{s}^{\rho, \nu}=C_{2}^{\rho}+C_{1}^{\rho}\left(I_{n_{1}^{\rho}}-A_{11}^{\rho, \nu}\right)^{-1} A_{12}^{\rho, \nu} \\
& D_{s}^{\rho, \nu}=C_{1}^{\rho}\left(I_{n_{1}^{\rho}}-A_{11}^{\rho, \nu}\right)^{-1} B_{1}^{\rho, \nu}
\end{aligned}
$$

and $\left(I_{n_{1}^{\rho}}-A_{11}^{\rho, \nu}\right)$ non-singular; the fast subsystem is defined as:

$$
\left\{\begin{array}{l}
x_{f}^{\rho}(k+1)=A_{11}^{\rho, \nu} x_{f}^{\rho}(k)+B_{1}^{\rho, \nu} u_{f}(k) \\
y_{f}(k)=C_{1}^{\rho} x_{f}^{\rho}(k) .
\end{array}\right.
$$

In Table 1, the eigenvalues $\varphi\left\{A^{\rho}\right\}$ of the original system (18) and the eigenvalues $\varphi\left\{A_{s}^{\rho}\right\}$ of the slow subsystem (20) are given for the 4-stands subsystem of a product from the Eisenhüttenstadt HSM database characterised by $w_{n}=1203 \mathrm{~mm}$ and $h_{n}=3.03 \mathrm{~mm}$. Notice that the time-scale separation justifies the use of the only slow dynamics for control design purposes. Moreover, the system is unstable (the first eigenvalue is greater than 1).

In the $n$-stands subsystem, the strip is connected to all the stands, and it is subject to a strong perturbation due to the coilbox vibrations. Hence, the main control task consists in guaranteeing a good quality of the rolled product, minimising the external perturbation. This phase takes the $90 \%-$ $95 \%$ of the whole rolling process duration and the system reaches the steady state before the switchings occur. 
In the tail end phase, traction is lost every time the strip leaves a stand. This increases the difficulties to guide the strip, which becomes free to move in all directions. Then, the priority of the control system is the safety of the system. Moreover, the tail end phase is very short and the switchings are very fast. Hence, dwell time conditions are not verified [19], [27]. This means that the stability of all subsystems is not a sufficient condition to guarantee the stability of the whole system. It is also necessary to verify that the switchings do not destabilise the system [12].

In the next section, a control law guaranteeing the asymptotic stability of the tail end phase is presented. To our knowledge, all the conditions to design a control law stabilising a switched system need a state vector with constant components and dimension. Nevertheless, in the HSM system, the components and dimension of the state vector change at each switching time. A possible solution consists in designing a robust control law stabilising each subsystem $\rho$ of (18) separately, $\forall \nu \in \Gamma$. The stability of the switched system will be verified a posteriori. In fact, the switching time depends on the rolled strip and must be estimated on-line. Then, the switched system stability condition has also to take into account an uncertainty in the switching time.

Since the LQ control has yielded good results for the $n$-stands subsystem, this approach is maintained also to the tail end phase. In [23], an alternative LMI solution for the LQ optimal control design is proposed. This approach has been extended to singularly perturbed systems in [5] and in [15] (for continuous and discrete-time systems, respectively). The advantage associated with the LMI formulation is the existence of several solvers that also provide solutions also in the case of high dimension problems. Moreover, the LMI-based controller can be directly extended to the uncertain systems, if $A_{11}^{\rho, \nu}$ is $\operatorname{Schur} \forall(\rho, \nu) \in \Sigma \times \Gamma$.

\section{Control design}

The first step consists in designing a robust control law which stabilises each subsystem $\rho$ of the tail end phase. Hence, for the moment, the effect of the switchings is not taken into account. The $\rho$ index is omitted and the obtained stability results will be applied to each subsystem $\rho$ separately. In the HSM system, due to actuators rate limits, only the slow subsystem can be controlled. Since $A_{11}^{\nu}$ is Schur, a reduced state-feedback gain controlling only the slow manifold stabilises the whole closed-loop system. 
Let choose the weighting matrix $R$ such that $R=R^{\prime}=G^{\prime} G \succ 0$. The following theorem designs a sub-optimal reduced controller stabilising the system (18) $\forall \nu \in \Gamma$. An upper bound of the performance degradation is given in [22].

Theorem 1. [15] If $A_{11}^{\nu}$ is Schur and there exist matrices $X_{s}=X_{s}^{\prime} \succ 0$, $W_{s}=W_{s}^{\prime} \succ 0$ and $S_{s}$ of appropriate dimension such that the LMI optimisation problem

$$
\min _{X_{s}, S_{s}, W_{s}} \operatorname{Tr}\left(X_{s}\right)
$$

under

$$
\left[\begin{array}{ccc}
X_{s} & C_{s}^{\nu} W_{s}+D_{s}^{\nu} S_{s} & G S_{s} \\
(\star)^{\prime} & W_{s} & 0 \\
(\star)^{\prime} & (\star)^{\prime} & W_{s}
\end{array}\right] \succeq 0
$$

and

$$
A_{s}^{\nu} W_{s}+W_{s} A_{s}^{\nu \prime}+B_{s}^{\nu} S_{s}+S_{s}^{\prime} B_{s}^{\nu \prime} \prec 0
$$

has a solution, then the reduced state-feedback control law

$$
u(k)=K\left[\begin{array}{l}
x_{1}(k) \\
x_{2}(k)
\end{array}\right]
$$

with $K=\left[\begin{array}{ll}0 & S_{s} W_{s}^{-1}\end{array}\right]$, guarantees the asymptotical stability of the closed-loop system (18), $\forall \nu \in \Gamma$.

The second step consists in verifying the stability of the switched system, $\forall(\rho, \nu) \in \Sigma \times \Gamma$. An uncertainty in the switching time must also be considered. Applying Theorem 1 to each subsystem $\rho$, we obtain $\mathcal{N}_{s}$ robust controller gains $K^{\rho}$ such that the closed-loop state matrix

$$
T_{\rho, \nu}=A^{\rho, \nu}+B^{\rho, \nu} K^{\rho}
$$

is Schur $\forall(\rho, \nu) \in \Sigma \times \Gamma$, where $A^{\rho, \nu}$ and $B^{\rho, \nu}$ are defined in (19). The change of basis

$$
z=E^{\rho-1} x^{\rho}
$$

can be applied for obtaining the same state vector $z, \forall \rho \in \Sigma$. Using matrices (27) $\forall(\rho, \nu) \in \Sigma \times \Gamma$, we obtain the closed-loop switched system in the polytopic form:

$$
z(k+1)=\mathcal{T}_{\sigma(k)} z(k)
$$


where $\left\{\mathcal{T}_{\rho}: \rho \in \Sigma\right\}$ is a family of matrices, $\sigma(k): \mathbb{N} \rightarrow \Sigma$ is the switching signal, which is assumed to be unknown a priori, and

$$
\mathcal{T}_{\sigma(k)}=\sum_{\rho=1}^{\mathcal{N}_{s}} \xi_{\rho}(k) \hat{\mathcal{T}}_{\rho}=\sum_{\rho=1}^{\mathcal{N}_{s}} \sum_{\nu=1}^{\mathcal{N}_{v}} \xi_{\rho}(k) \lambda_{\rho, \nu}(k) \bar{T}_{\rho, \nu}
$$

with $\bar{T}_{\rho, \nu}=E^{\rho-1} T_{\rho, \nu} E^{\rho}, \xi_{\rho}(k): \mathbb{N} \rightarrow\{0,1\}, \sum_{\rho=1}^{\mathcal{N}_{s}} \xi_{\rho}(k)=1$ and $\lambda_{\rho, \nu}(k) \geq 0$, $\sum_{\rho=1}^{\mathcal{N}_{v}} \lambda_{\rho, \nu}(k)=1, \forall(\rho, \nu) \in \Sigma \times \Gamma$.

The following theorem gives a condition to verify the asymptotic stability of an autonomous switched system in the form

$$
z(k+1)=A^{\sigma(k)} z(k)
$$

using the concept of dwell time $\Delta \in \mathbb{N}$. Consider two successive switching times $l_{q}$ and $l_{q+1}$ satisfying $l_{q+1}-l_{q}=\Delta_{q} \geq \Delta \geq 1, \forall q \in \mathbb{N}$. Moreover, consider that for $k \in\left[l_{q}, l_{q+1}\right)$ the system is in the subsystem corresponding to $\sigma(k)=\rho \in \Sigma$ and that when a switching occurs, for $k=l_{q+1}$, the system jumps to the subsystem corresponding to $\sigma(k)=\rho_{+} \in \Sigma$.

Theorem 2. [6] If there exist $\mathcal{N}_{s}$ matrices $P_{\rho}=P_{\rho}^{\prime} \succ 0$ of appropriate dimensions such that the LMIs

$$
\begin{gathered}
A_{\rho}^{\prime} P_{\rho} A_{\rho}-P_{\rho} \prec 0, \forall \rho \in \Sigma, \\
A_{\rho}^{\Delta^{\prime}} P_{\rho_{+}} A_{\rho}^{\Delta}-P_{\rho} \prec 0, \forall\left(\rho, \rho_{+} \neq \rho\right) \in \Sigma \times \Sigma
\end{gathered}
$$

are verified, then the system (31) is asymptotically stable for $\Delta_{q} \geq \Delta \geq 1$.

Theorem 2 does not require a Lyapunov function uniformly decreasing at each switching time, under the assumption of $\Delta \geq 1$. This fact reduces the conservatism. In the HSM system case, only $n-1$ switchings can occur. Then, it is not necessary to verify the LMI $(33) \forall\left(\rho, \rho_{+} \neq \rho\right) \in \Sigma \times \Sigma$. Moreover, the minimum dwell time $\Delta^{\rho}$ is known $\forall \rho \in \Sigma$. Nevertheless, in order to prove the stability of system (29), a condition taking into account the 
uncertain parameters $\forall \nu \in \Gamma$ and an uncertainty $\tau^{\rho} \in \Omega^{\rho}=\left\{-\mathcal{N}_{\tau^{\rho}}, \ldots, \mathcal{N}_{\tau^{\rho}}\right\}$ in the switching time is needed.

Consider three successive switching times $l_{q-1}, l_{q}$ and $l_{q+1}$. For $k \in$ $\left[l_{q-1}, l_{q}\right)$ the system is in the subsystem corresponding to $\left(\rho_{-}, \nu_{-}, \tau^{\rho_{-}}\right) \in$ $\Sigma \times \Gamma \times \Omega^{\rho_{-}}$, for $k \in\left[l_{q}, l_{q+1}\right)$ the system is in the subsystem corresponding to $\left(\rho, \nu, \tau^{\rho}\right) \in \Sigma \times \Gamma \times \Omega^{\rho}$ and, for $k=l_{q+1}$, the system jumps to the subsystem corresponding to $\left(\rho_{+}, \nu_{+}, \tau^{\rho_{+}}\right) \in \Sigma \times \Gamma \times \Omega^{\rho_{+}} . l_{q-1}, l_{q}$ and $l_{q+1}$ satisfy $l_{q}-l_{q-1}=\Delta_{q-1}^{\rho_{-}} \geq \Delta^{\rho_{-}} \geq 1, l_{q+1}-l_{q}=\Delta_{q}^{\rho} \geq \Delta^{\rho} \geq 1, \forall q \in \mathbb{N}$, where $\Delta^{\rho}$ is the dwell time of the subsystem $\rho$. We assume $\mathcal{N}_{\tau^{\rho}}+\mathcal{N}_{\tau^{\rho+}}<\Delta^{\rho}$. Hence, the sub-

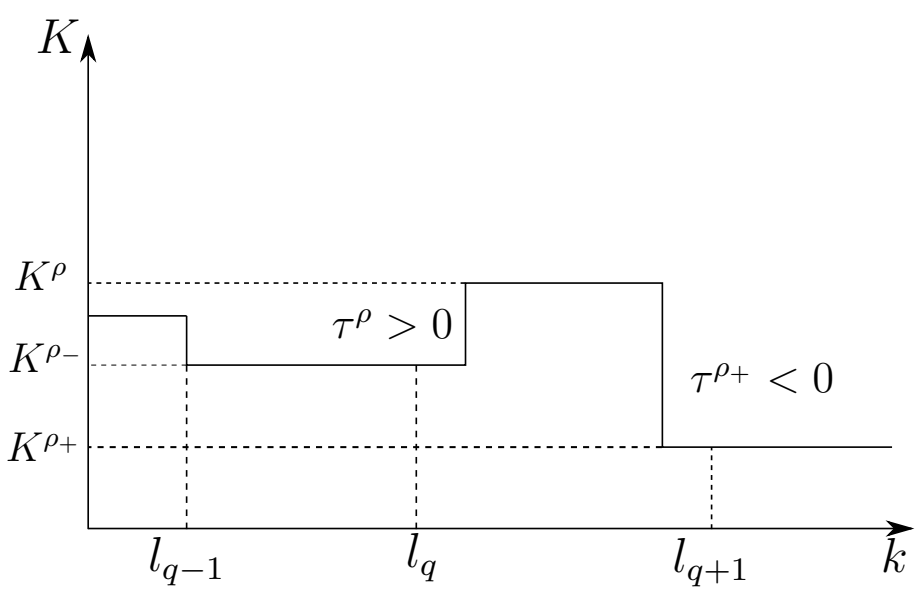

Figure 2: Controller switchings

system $\rho$ is controlled by the wrong gain $K^{\rho_{-}}$for a time $t \in\left(k T_{s},\left(k+\tau^{\rho}\right) T_{s}\right)$ if $\tau^{\rho}>0$, with $\bar{T}_{\rho_{-}, \nu}=E^{\rho-1}\left(A^{\rho, \nu}+B^{\rho, \nu} K^{\rho_{-}}\right) E^{\rho}$. Furthermore, the subsystem $\rho$ is controlled by the wrong gain $K^{\rho_{+}}$for a time $t \in\left(k T_{s},\left(k-\tau^{\rho_{+}}\right) T_{s}\right)$ if $\tau^{\rho_{+}}<0$, with $\bar{T}_{\rho_{+}, \nu}=E^{\rho-1}\left(A^{\rho, \nu}+B^{\rho, \nu} K^{\rho_{+}}\right) E^{\rho}$ (Fig. 2).

Let the transition matrix $Q_{\pi, \Delta_{q}^{\rho}}$, which represents the system evolution for $k \in\left[l_{q}, l_{q+1}\right)$. Its value depends on the sign of $\tau^{\rho}$ and $\tau^{\rho_{+}}$:

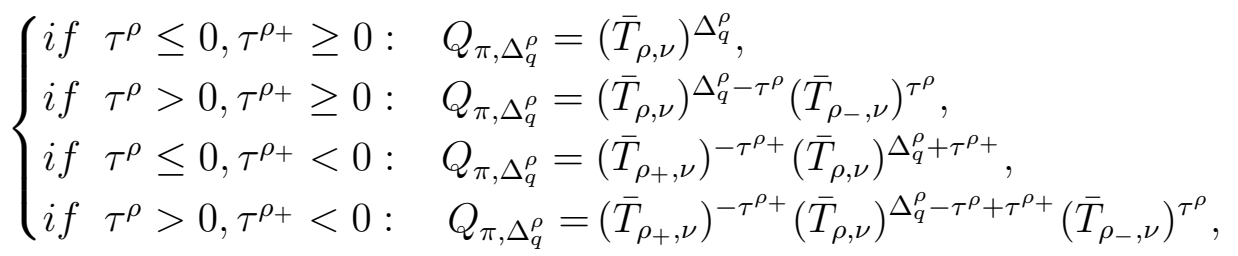

with $\pi=\rho, \rho_{-} \neq \rho, \rho_{+} \neq \rho, \nu, \nu_{+}, \tau^{\rho}, \tau^{\rho_{+}} \in \Pi=\Sigma \times \Sigma \times \Sigma \times \Gamma \times \Gamma \times \Omega^{\rho} \times \Omega^{\rho_{+}}$. The following theorem gives a generalisation of Theorem 2 for uncertain 
switched systems with an uncertainty in the switching time.

Theorem 3. Consider the uncertain switched system (29) and an uncertainty in the control signal switching $\tau^{\rho} \in \Omega^{\rho}$. If there exist $\mathcal{N}_{s} \times \mathcal{N}_{v}$ matrices $P_{\rho, \nu}=P_{\rho, \nu}{ }^{\prime} \succ 0$ of appropriate dimensions such that the LMIs

$$
\begin{gathered}
\bar{T}_{\rho, \nu}^{\prime} P_{\rho, \nu} \bar{T}_{\rho, \nu}-P_{\rho, \nu} \prec 0, \forall(\rho, \nu) \in \Sigma \times \Gamma, \\
Q_{\pi, \Delta^{\rho}}^{\prime} P_{\rho_{+}, \nu_{+}} Q_{\pi, \Delta^{\rho}}-P_{\rho, \nu} \prec 0, \forall \pi \in \Pi
\end{gathered}
$$

are verified, then the switched system (29) is asymptotically stable for $\Delta_{q}^{\rho} \geq$ $\Delta^{\rho} \geq 1, \forall\left(\rho, \nu, \tau^{\rho}\right) \in \Sigma \times \Gamma \times \Omega^{\rho}$.

Proof 1. Let the parameter dependent Lyapunov function

$$
V(k)=z(k)^{\prime} \sum_{\rho=1}^{\mathcal{N}_{s}} \sum_{\nu=1}^{\mathcal{N}_{v}} \xi_{\rho}(k) \lambda_{\rho, \nu}(k) P_{\rho, \nu} z(k)
$$

where $P_{\rho, \nu}=P_{\rho, \nu}^{\prime} \succ 0$. The system (29) is asymptotically stable if the difference of the Lyapunov function $\mathcal{L}(k)=V(k+1)-V(k)$ satisfies the inequality

$$
\mathcal{L}(k)=z(k)^{\prime}\left(\mathcal{T}(k)^{\prime} \mathcal{P}_{+}(k) \mathcal{T}(k)-\mathcal{P}(k)\right) z(k) \prec 0
$$

where

$$
\begin{gathered}
\mathcal{P}(k)=\sum_{\rho=1}^{\mathcal{N}_{s}} \sum_{\nu=1}^{\mathcal{N}_{v}} \xi_{\rho}(k) \lambda_{\rho, \nu}(k) P_{\rho, \nu} \\
\mathcal{P}_{+}(k)=\sum_{\rho=1}^{\mathcal{N}_{s}} \sum_{\nu=1}^{\mathcal{N}_{v}} \xi_{\rho}(k+1) \lambda_{\rho, \nu}(k+1) P_{\rho, \nu}=\sum_{\rho_{+}=1}^{\mathcal{N}_{s}} \sum_{\nu_{+}=1}^{\mathcal{N}_{v}} \xi_{\rho_{+}}(k) \lambda_{\rho_{+}, \nu_{+}}(k) P_{\rho_{+}, \nu_{+}}
\end{gathered}
$$

$\forall(\rho, \nu) \in \Sigma \times \Gamma$ and $\forall\left(\rho_{+}, \nu_{+}\right) \in \Sigma \times \Gamma\left[\right.$ [7]. From (35), $\forall k \in\left[l_{q}, l_{q+1}\right)$ the Lyapunov function $v(z(k))=z(k)^{\prime} P_{\rho, \nu} z(k)$ satisfies

$$
v(z(k+1))<v(z(k)) .
$$

Hence, there exist scalars $\alpha \in(0,1)$ and $\beta>0$ such that

$$
\|z(k)\|_{2}^{2} \leq \beta \alpha^{k-l_{q}} v\left(z\left(l_{q}\right)\right),
$$


$k \in\left[l_{q}, l_{q+1}\right)$. Moreover, from (34), when $\tau^{\rho}>0$ and $\tau^{\rho_{+}}<0$, we have $Q_{\pi, \Delta_{q}^{\rho}}=\left(\bar{T}_{\rho_{+}, \nu}\right)^{-\tau^{\rho_{+}}}\left(\bar{T}_{\rho, \nu}\right)^{\Delta_{q}^{\rho}-\tau^{\rho}+\tau^{\rho_{+}}}\left(\bar{T}_{\rho_{-}, \nu}\right)^{\tau^{\rho}}$. Hence, using (36) we obtain:

$$
\begin{aligned}
v\left(z\left(l_{q+1}\right)\right) & =z\left(l_{q+1}\right)^{\prime} P_{\rho_{+}, \nu_{+}} z\left(l_{q+1}\right) \\
& =z\left(l_{q}\right)^{\prime}\left(\bar{T}_{\rho_{+}, \nu}^{-\tau_{+}} \bar{T}_{\rho, \nu}^{\Delta_{q}^{\rho}-\tau^{\rho}+\tau^{\rho_{+}}} \bar{T}_{\rho_{-}, \nu}^{\tau^{\rho}}\right)^{\prime} P_{\rho_{+}, \nu_{+}} \bar{T}_{\rho_{+}, \nu}^{-\tau_{+}{ }^{\rho_{+}}} \bar{T}_{\rho, \nu}^{\Delta_{q}^{\rho}-\tau^{\rho}+\tau^{\rho_{+}}} \bar{T}_{\rho_{-}, \nu}^{\tau^{\rho}} z\left(l_{q}\right) \\
& <z\left(l_{q}\right)^{\prime}\left(\bar{T}_{\rho_{-}, \nu}^{-\tau^{\rho}} \bar{T}_{\rho, \nu}^{\Delta_{q}^{\prime}-\Delta^{\rho}} \bar{T}_{\rho_{-}, \nu}^{\tau^{\rho}}\right)^{\prime} P_{\rho, \nu} \bar{T}_{\rho_{-}, \nu}^{-\tau^{\rho}} \bar{T}_{\rho, \nu}^{\Delta_{q}^{\rho}-\Delta^{\rho}} \bar{T}_{\rho_{-}, \nu}^{\tau^{\rho}} z\left(l_{q}\right) \\
& \leq z\left(l_{q}\right)^{\prime}\left(\bar{T}_{\rho_{-}, \nu}^{\tau^{\rho}-\tau^{\rho}}\right)^{\prime} P_{\rho, \nu} \bar{T}_{\rho_{-}, \nu}^{\tau^{\rho}-\tau^{\rho}} z\left(l_{q}\right) \\
& =z\left(l_{q}\right)^{\prime} P_{\rho, \nu} z\left(l_{q}\right)=v\left(z\left(l_{q}\right)\right) .
\end{aligned}
$$

The non-strict inequality holds because $\Delta_{q}^{\rho}-\Delta^{\rho} \geq 0$ and $\bar{T}_{\rho, \nu}$ is Schur. Then

$$
\left(\bar{T}_{\rho, \nu}^{\Delta_{q}^{\rho}-\Delta^{\rho}}\right)^{\prime} \bar{T}_{\rho_{-, \nu}}^{-\tau^{\rho^{\prime}}} P_{\rho, \nu} \bar{T}_{\rho_{-}, \nu}^{-\tau^{\rho}} \bar{T}_{\rho, \nu}^{\Delta_{q}^{\rho}-\Delta^{\rho}} \preceq \bar{T}_{\rho_{-}, \nu}^{-\tau^{\rho^{\prime}}} P_{\rho, \nu} \bar{T}_{\rho_{-, \nu}^{-\tau^{\rho}}}
$$

$\forall(\rho, \nu) \in \Sigma \times \Gamma$. The relation $v\left(z\left(l_{q+1}\right)\right)<v\left(z\left(l_{q}\right)\right)$ is verified also for the other cases of (34). In order to see it, it is sufficient to substitue the right value of $Q_{\pi, \Delta_{q}^{\rho}}$ in (43). Hence, given the initial condition of (29) $z(0)=z_{0}$, there exists $\mu \in(0,1)$ such that

$$
v\left(z\left(l_{q}\right)\right) \leq \mu^{q} v\left(z_{0}\right), \forall q \in \mathbb{N} .
$$

Finally, (42) and (45) imply that the system (29) is asymptotically stable.

Remark 1. In order to verify the LMIs (35)-(36) of Theorem 3, $\mathcal{N}_{s} \mathcal{N}_{v}+$ $\mathcal{N}_{s}\left(\mathcal{N}_{s}-1\right)^{2} \mathcal{N}_{v}^{2}\left(\mathcal{N}_{\tau^{\rho}}+1\right)^{2}$ possible combinations have to be considered, in the general case. Nevertheless, in the HSM system case, only $n-1$ switchings can occur. Moreover, since uncertain parameters are constant for each product, switchings are possible only between subsystems with the same $\nu$. Then, only $\mathcal{N}_{s} \mathcal{N}_{v}+(n-1) \mathcal{N}_{v}\left(\mathcal{N}_{\tau^{\rho}}+1\right)^{2}$ LMIs have to be verified.

\section{Numerical results}

In this section, a robust discrete-time controller is designed in order to stabilise the tail end phase of the Eisenhüttenstadt HSM $(n=5)$. In order to obtain the physical characteristics of the products corresponding to the vertices, compute the state matrices and the controller gains of each family, the Matlab toolbox $R S C T$ has been used [14]. The software also allows to verify the stability of the switched system. The LMI problems of Theorem 1 
and 3 are solved using the free LMI solver SeDuMi [26] and the free Matlab toolbox $Y A L M I P$, which provides a simple interface for the most popular LMI solvers [13].

The robust controller gain $K^{\rho}$ guaranteeing the stability of each subsystem $\rho \in \Sigma$ can be designed using Theorem 1. Weighting matrices are:

$$
R^{4}=R_{0}^{4}\left[\begin{array}{ccccc}
1 & 0 & 0 & 0 & 0 \\
0 & 1 & 0 & 0 & 0 \\
0 & 0 & 1 & 0 & 0 \\
0 & 0 & 0 & 1 & 0 \\
0 & 0 & 0 & 0 & 0.1
\end{array}\right], R^{3}=R_{0}^{3}\left[\begin{array}{cccc}
1 & 0 & 0 & 0 \\
0 & 1 & 0 & 0 \\
0 & 0 & 1 & 0 \\
0 & 0 & 0 & 0.1
\end{array}\right], R^{2}=R_{0}^{2}\left[\begin{array}{ccc}
1 & 0 & 0 \\
0 & 1 & 0 \\
0 & 0 & 0.1
\end{array}\right] .
$$

$R_{0}^{4}, R_{0}^{3}$ and $R_{0}^{2}$ have been set in order to prevent the saturation of the control signal. Notice that the value corresponding to the last stand is smaller than the others. The main reason of this choice is that the strip is very thin in the last stand. Then, even a little correction of the stand tilting can be dangerous for the system stability. The idea consists in controlling only the first $n-1$ stands. This means that the last subsystem, the 1-stands subsystem, is in open-loop. This strategy works well because the $Z$ value is very little when the last switching occurs, because of the controller action in the previous subsystems. Moreover, the law of matter conservation $\left(h_{j-1} s_{j-1}=h_{j} s_{j}\right)$ has to be respected. Hence, the strip is very fast in the last stands. This means that the dwell time is very short. Thus, the strip off-centre cannot increase too much.

Given the controller gains and the dwell time $\Delta^{\rho}$, Theorem 3 provides a sufficient condition for the stability of the switched system $\forall \tau^{\rho} \in \Omega^{\rho}$. We found a solution for $\mathcal{N}_{\tau}^{\rho} \leq 4, \forall \rho \in \Sigma$. Since $T_{s}=50 \mathrm{msec}$, the stability of the system is guaranteed for a maximum uncertainty of $\pm 200 \mathrm{msec}$ in the switching time. From practical experience, this constraint can be respected.

The following simulations have been done using the nonlinear HSM model given in [3]. Let a product with $w=903 \mathrm{~cm}$ and $h_{n}=4.33 \mathrm{~cm}$.

In Fig. 3, the $Z$ evolution in output to each stand is shown. No delay in the control signal has been considered. The value of $Z_{j}$ goes to zero when the strip leaves the $j^{\text {th }}$ stand. The solid line represents the $Z$ evolution when the system is controlled by the robust controller gain computed using Theorem 1. The dashed line shows the $Z$ evolution when the system is controlled by a LQ controller gain designed using average state matrices. This method has been proposed in [3] for the $n$-stands subsystem. The dotted line corresponds 
to the $Z$ evolution when the system works in open-loop.

We can see that the robust controller is able to keep the $Z$ value near to zero during all the rolling process. Otherwise, the average LQ controller limits the $Z$ value for the first four stands but induces an oscillatory behaviour on the strip. Then, when the strip leaves stand 4 , the $Z$ value increases very quickly. This situation can be very dangerous for the system. In the open loop case, the $Z$ value begins to increase when the strip leaves the first stand $\left(N_{s}=1613\right)$. Notice that in stand 4 and 5 a saturation occurs. This means that the strip is crashing against the side guides because of the elevated $Z$. The result is a decrease of the product quality and, in the worst case, the damage of the rolls. In Fig. 4, an uncertainty in the switching signal is introduced. In the Eisenhüttenstadt case, the switching time can be estimated online, with an error that has the same sign $\forall \rho \in \Sigma$. Here, the case $\tau^{\rho} \geq 0$ is presented. This means that the controller switches to the $\rho$ stands subsystem $\tau^{\rho}$ times after the strip left the stand. Three different cases are showed: $\left(\tau=\tau^{4}=\tau^{3}=\tau^{2}=4\right)($ solid line $),\left(\tau=\tau^{4}=\tau^{3}=\tau^{2}=8\right)$ (dashed line), $\left(\tau=\tau^{4}=\tau^{3}=\tau^{2}=12\right)$ (dotted line). Although theoretically the system is stable only for $\tau \leq 4$, notice that until $\tau=8$ the $Z$ value is kept near to zero. Nevertheless, when $\tau=12$ the controller performances decrease and the strip almost crashes against the side-guides. In this case, the $Z$ value increases in the opposite side of the open-loop case.

In Fig. 5, we consider the same delay using the average LQ controller. We can see that this kind of controller does not accept uncertainties on the switching time. The system is unstable and the strip crashes against the side guides in each case.

\section{Conclusion}

In this article, a discrete-time robust control has been proposed for the tail end phase of the HSM system, which has been modelled as an uncertain switched system in the singular perturbation form. Also uncertainties in the switching time have been considered.

Simulation results concerning the Eisenhüttenstadt ArcelorMittal HSM showed that the strip off-centre can be reduced, improving the reliability of the industrial process. The proposed approach can be adapted to other plants. 


\section{Acknowledgements}

This work has been supported by grants from "la région Lorraine, France" and ArcelorMittal Maizières Research.

\section{References}

[1] S. Boyd, L.E. Ghaoui, E. Feron, and V. Balakrishnan. Linear Matrix Inequalities in system and control theory. Society for Industrial and Applied Mathematics, 1994.

[2] I. S. Choi, J. A. Rossiter, J. S. Chung, and P. J. Fleming. An MPC strategy for hot rolling mills and applications to strip threading control problems. In IFAC world congress, 2008.

[3] J. Daafouz, R. Bonidal, C. Iung, P. Szczepanski, N. Naumann, and U. Koschack. New steering control at EKO Stahl finishing mill. In The Iron 65 Steel Technology Conference and Exposition, 2008.

[4] Y. Furukawa, S. Fujii, and H. Taoka. Application of steering control in hot strip mill. Tetsu-to-Hagane (Journal of the Iron and Steel Institute of Japan), 78(8):141-144, 1992.

[5] G. Garcia, J. Daafouz, and J. Bernussou. The infinite time near optimal decentralized regulator problem for singularly perturbed systems: a convex optimization approach. Automatica, 38(8):1397-1406, 2002.

[6] J.C. Geromel and P. Colaneri. Stability and stabilization of discrete time switched systems. International Journal of Control, 79(7):719-728, 2006.

[7] L. Hetel, J. Daafouz, and C. Iung. Robust stability analysis and control design for switched uncertain polytopic systems. In 5th IFAC Workshop on Robust Control, 2006.

[8] H. Kando and T. Iwazumi. Multirate digital control design of an optimal regulator via singular perturbation theory. International Journal of Control, 44(6):1555-1578, 1986.

[9] T. Kimura and M. Tagawa. Automatic steering control of strip rolling mills. The Hitachi Review, 65(2):25-30, 1983. 
[10] P. Kokotovic, H.K. Khalil, and J. O'Reilly. Singular perturbation methods in control: analysis and design. Academic Press, 1986.

[11] H. Kuwano and N. Takahashi. Sensor-type automatic steering control system for rolling mill $-1^{\text {st }}$ report. Ishikawajima-Harima Engineering Review, 26(1):35-40, 1986.

[12] D. Liberzon. Switching in systems and control. Birkhäuser, 2003.

[13] J. Löefberg. YALMIP : A toolbox for modeling and optimization in MATLAB. In IEEE international symposium on computer aided control systems design, 2004.

[14] I. Malloci, R. Bonidal, P. Szczepanski, J. Daafouz, and C. Iung. Robust Steering Control Toolbox. Internal Rapport ArcelorMittal, 2008.

[15] I. Malloci, J. Daafouz, C. Iung, and R. Bonidal. A LMI solution to the LQ problem for discrete time singularly perturbed systems. In European Control Conference, 2009.

[16] I. Malloci, J. Daafouz, C. Iung, R. Bonidal, and P. Szczepanski. Robust steering control of hot strip mill. Submitted to Pubblication, 2009.

[17] Y. Marushita, H. Ikeda, K. Yano, and S. Shindo. Advanced control method of steering on the hot rolling mill. In 5th IFAC Automation in Mining, Mineral, and Metal Processing, 2001.

[18] H. Matsumoto and K. Nakajima. Automatic side-walk control in hot strip mill. In Japanese joint conf. for the technology of plastic, 1980.

[19] A.S. Morse. Supervisory control of families of linear set-point controllers - part 1: Exact matching. IEEE Transactions on Automatic Control, 41:1413-1431, 1996.

[20] M. Okada, K. Murayama, Y. Anabuki, and Y. Hayashi. VSS control of strip steering for hot rolling mills. In IFAC world congress, 2005.

[21] Y. Okamura and I. Hoshino. State feedback control of the strip steering for aluminum hot rolling mill. Control Engineering Practice, 5(8):10351042, 1997. 
[22] H. Othman, N. Khraishi, and M. S. Mahmoud. Discrete regulators with time-scale separation. IEEE Transactions on Automatic Control, 30(3):293- 297, 1985.

[23] P.L.D. Peres and J.C. Geromel. An alternate numerical solution to the linear quadratic problem. IEEE Transactions on Automatic Control, 39(1):198-202, 1994.

[24] H. Saitoh and T. Sakimoto. Development of advanced steering control of hot strip mill in steel production process. 1992.

[25] M.J. Steeper and G.D. Park. Development of steering control system for reversing hot mills. Iron and steel engineer, 75(11):21-24, 1998.

[26] J.F. Sturm. Using SeDuMi 1.02, a Matlab Toolbox for optimisation over symmetric cones. Optimization Methods and Software, 11-12:625653, 1999.

[27] G. Zhai, B. Hu, K. Yasuda, and A.N. Michel. Stability analysis of switched systems with stable and unstable subsystems: An average dwell time approach. International Journal of Systems Science, 32(8):1055-1061, 2001. 
stand 1
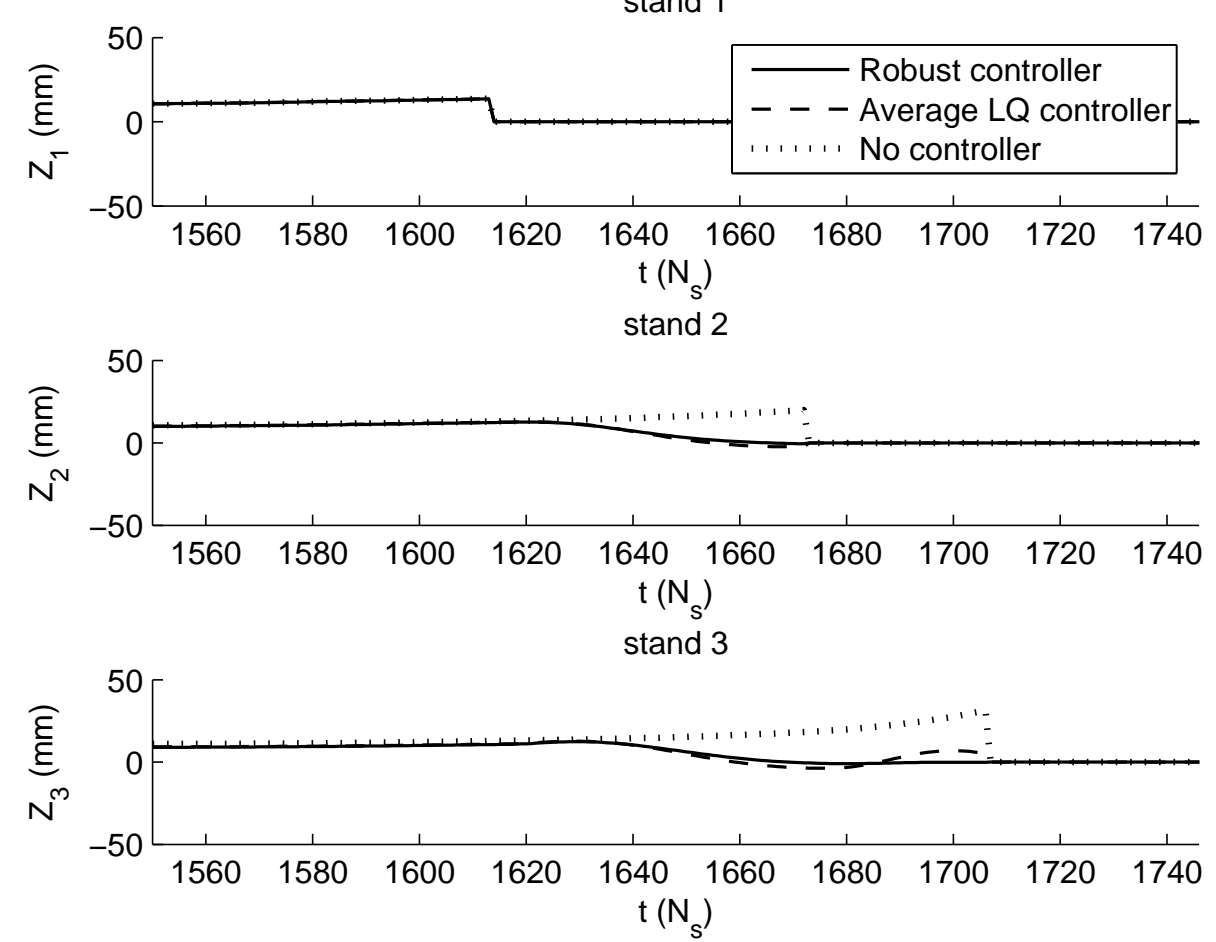

stand 4

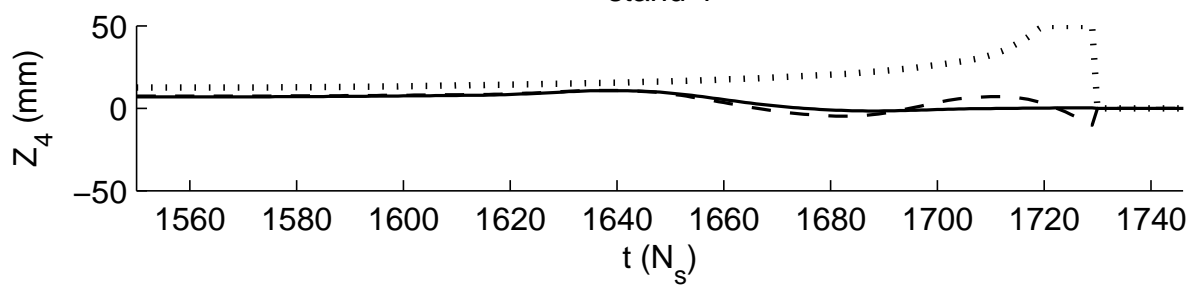

stand 5

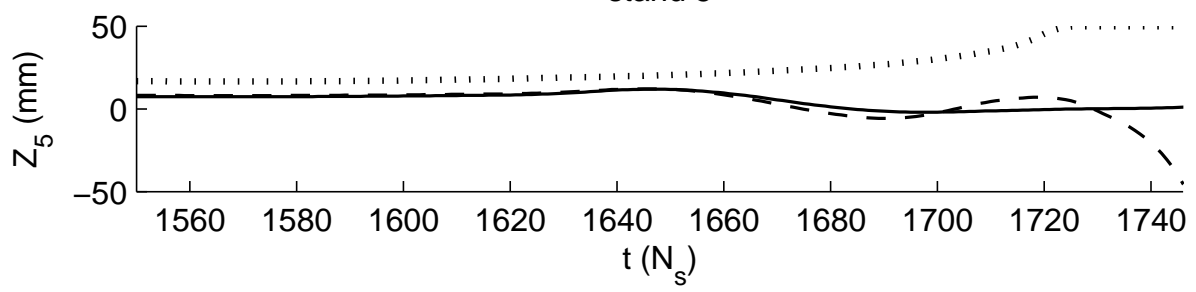

Figure 3: Strip off-centre evolution: comparison 

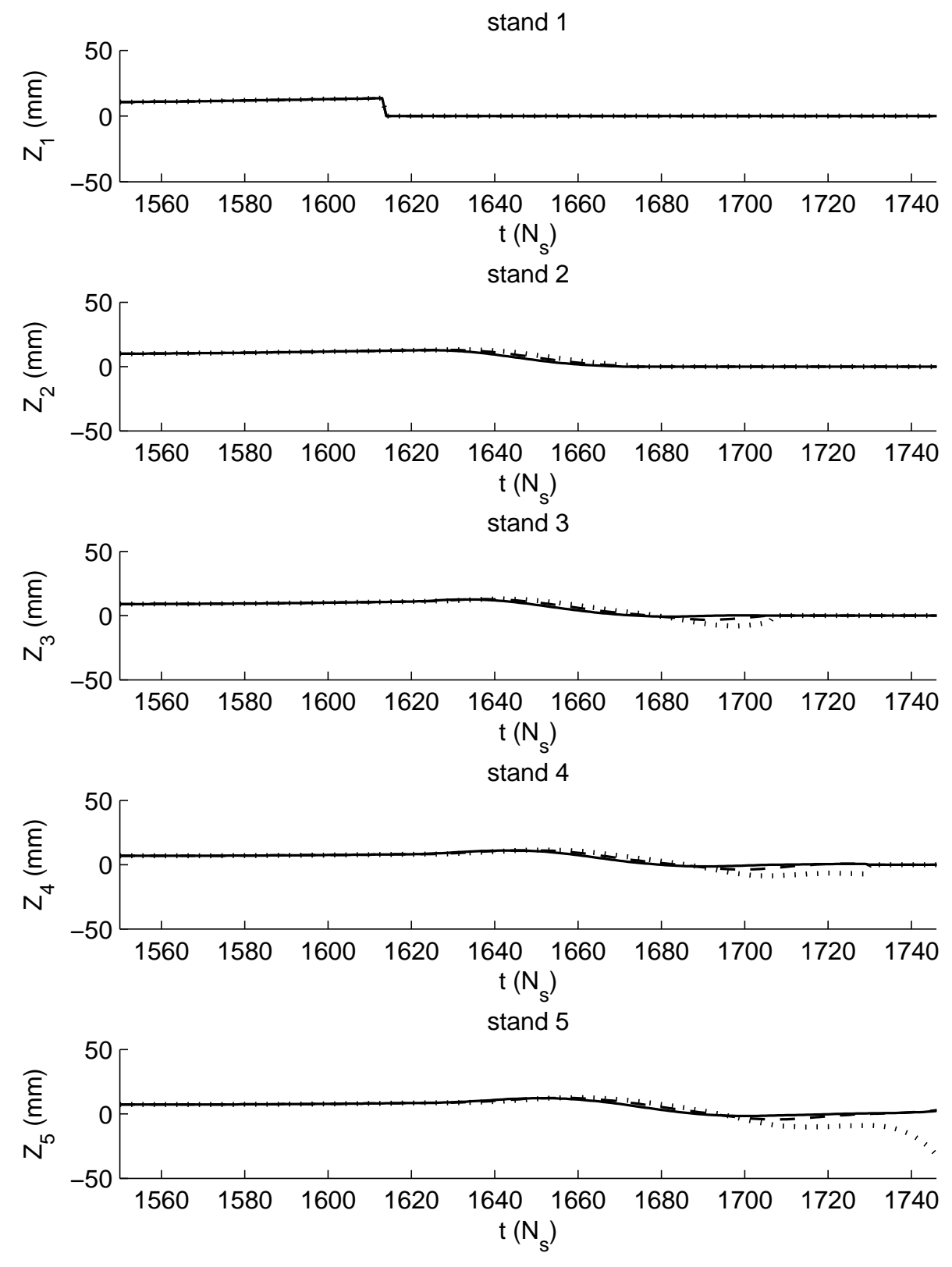

Figure 4: Strip off-centre evolution with delay in the robust controller switchings 

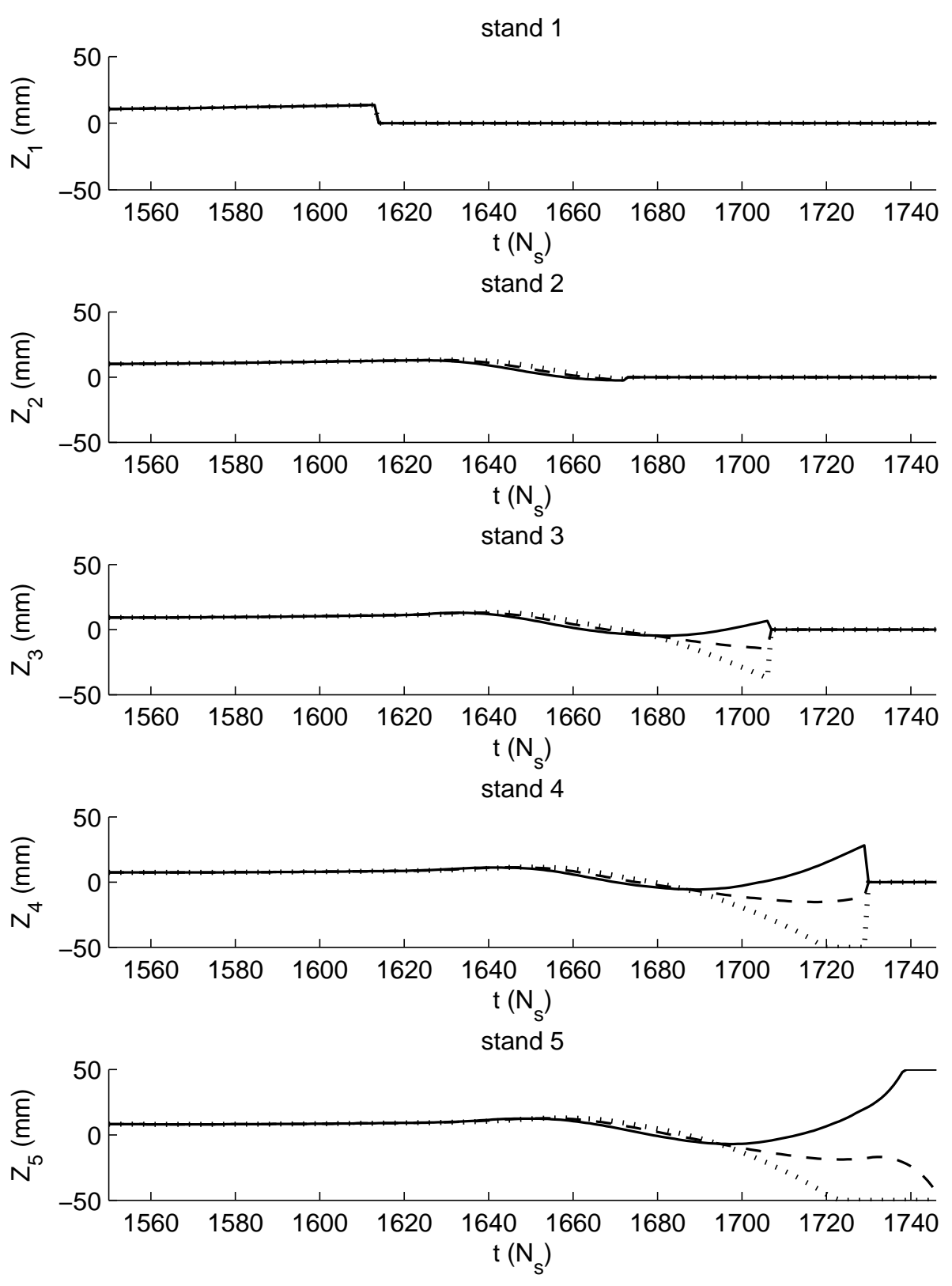

Figure 5: Strip off-centre evolution with delay in the average LQ controller switchings 